\section{PWE-238 IMMUNISATION PRACTICES IN PATIENTS WITH IBD-A WAKE UP CALL}

doi:10.1136/gutjnl-2012-302514d.238

R Muhammad, * C L Y Wong, J K Limdi. Department of Gastroenterology, Pennine Acute Hospitals NHS Trust, Greater Manchester, UK

Introduction Patients with inflammatory bowel disease on immunosuppressant therapy may have an increased risk of opportunistic infections. We reviewed our practice in 150 consecutive patients attending our IBD clinics to see if our practice was in line with recommendations by the European Crohn's and Colitis Organisation and the American College of Gastroenterology.

Methods Patients were considered immunosuppressed if they were on doses of prednisolone $>20 \mathrm{mg}$ /day or equivalent for 2 weeks or more, ongoing treatment with effective doses of 6-MP/Azathioprine, Methotrexate, Infliximab and Adalimumab or had these agents discontinued within 3 months. Data were collected from electronic records, clinical case notes and pathology results database review. Up to date and childhood immunisations including previous history of Varicella and Zoster infections was obtained from the primary care physicians.

Results 150 IBD patients (Crohn's disease $n=80$, Ulcerative colitis $\mathrm{n}=70$ ) were included; 79 were female. The median age was 51 and median disease follow-up was 23 years. 29 patients (19.3\%) were not on any treatment at the time of the audit, 19 (65.5\%) out of these 29 patients had therapy with glucocorticoid, thiopurine or biologics previously. 52 patients $(34.7 \%)$ were on a 5 ASA and 71 patients $(47.3 \%)$ were on effective immunosuppression as defined above. These included 26 patients on thiopurines (36.6\%), 3 on Methotrexate $(4.2 \%), 13$ on Infliximab (18.3\%), 12 on thiopurines + Infliximab (16.9\%), 1 on Methotrexate + Infliximab (1.41\%), four patients on Adalimumab (5.6\%), 3 on Adalimumab + thiopurines $(4.2 \%), 7$ on glucocorticoids alone $(9.9 \%)$ and 2 on Infliximab + Prednisolone $(2.8 \%)$. Immunisation history was taken in 29 out of the 150 patients (19.3\%). Among the patients on immunosuppressive therapy $29(40.8 \%)$ had chest radiographs performed, 16 $(22.5 \%)$ were tested for Hepatitis B, 16 (22.5\%) for Hepatitis C, 5 (7.0\%) for HIV and 4 (5.6\%) were tested for varicella titres. Cervical smears were performed in $14(19.7 \%)$ out of 25 women above the age of 25 . Of the 71 patients on immunosuppressants, $31(43.7 \%)$ had no screening tests. Immunisation was carried out for influenza in 42 patients $(59.1 \%), 30$ (42.3\%) for tetanus, 25 (35.2\%) for diphtheria, $12(16.9 \%)$ for pneumococcus, 7 (9.9\%) for meningococcus, 5 (7.0\%) for Hepatitis B, 4 (5.6\%) HPV and 11 (15.5\%) for MMR.

Conclusion Our current practice was not in line with recent recommendations and probably reflective of experience at other centres. IBD physicians must work in collaboration with primary care providers to ensure appropriate screening and vaccination in this vulnerable group. We have taken appropriate steps to ensure prompt screening of patients through a newly designed proforma.

Competing interests None declared

\section{PWE-239 THE ACCURACY OF PRE BONE MINERAL DENSITY FRAX SCORES IN PREDICTING NEED FOR TREATMENT IN PATIENTS WITH INFLAMMATORY BOWEL DISEASE AT RISK OF OSTEOPOROSIS}

doi:10.1136/gutjnl-2012-302514d.239

R 0 Butcher,* T Law, J K Limdi. Department of Gastroenterology, Pennine Acute Hospitals, Manchester, UK

Introduction Patients with Inflammatory Bowel disease (IBD) are at increased risk of Osteoporosis. Dual-Emission x-ray absorptiometry
(DEXA) scans are frequently used to assess for osteoporosis and guide the need for preventative therapy. The WHO fracture risk assessment web tool can be used with or without bone mineral density (BMD) to calculate a FRAX score (10-year probability of major osteoporotic fracture and hip fracture). Patients are assigned, based on their pre-BMD FRAX outcome into low, intermediate (requiring $\mathrm{BMD}$ measurement) and high (requiring preventative therapy) risk groups in accordance with guidelines from the National Osteoporosis Guidelines Group (NOGG). Our study aimed to assess the accuracy of Pre-BMD FRAX scores in predicting need for preventative therapy in an IBD cohort.

Methods We conducted a retrospective review of 95 patients who had undergone an index DEXA scan while attending IBD clinics at our hospital between 2007 and 2011. Clinical data including demographics, disease characteristics and therapy were obtained from case note and electronic patient record review. Pre and postBMD FRAX assessment scores were calculated retrospectively. All IBD patients were considered to have secondary osteoporosis as a risk factor for FRAX calculation. Fifteen patients were excluded as they had previously received bisphosphonates. Pre-BMD FRAX risk status was compared with Post-BMD FRAX outcome to identify patients requiring and not requiring therapy.

Results 80 patients (female 56) with a median age of 53.8 and mean disease duration of 14.1 years were analysed. 52 (65\%) patients had Crohn's disease, 27 (34\%) patients ulcerative colitis and one patient had IBD-type unclassified (IBDU). Pre-BMD FRAX risk status (low and intermediate/high risk) had a sensitivity of 100\% (95\% CI 87.9 to 100$)$, in correctly identifying 28 out of 28 patients as needing treatment. Specificity was low at $31 \%$ (95\% CI 19.9 to 44.3 ) identifying only 16 out of 52 patients as not needing treatment. The positive predictive value was low (44\%; $95 \%$ CI 32.3 to 55.9 ) with 28 out of 64 patients correctly identified as needing treatment but the negative predictive value was high $(100 \% ; 95 \%$ CI 80.6 to 100$)$ as all 16 patients were correctly identified as not needing treatment Specificity and PPV results were influenced by the intermediate risk Pre-BMD FRAX sub-group of which 33 (73\%) required lifestyle advice and $12(27 \%)$ required preventative therapy following postBMD FRAX assessment.

Conclusion In IBD patients, pre-BMD FRAX risk status is sensitive at predicting need for osteoporosis treatment and can help guide clinical decision making potentially avoiding DEXA scanning in IBD sub-groups.

Competing interests None declared.

\section{PWE-240 INCIDENTAL DIAGNOSIS OF INFLAMMATORY BOWEL DISEASE IN A BRITISH BOWEL CANCER SCREENING COHORT: A MULTI-CENTRE STUDY}

doi:10.1136/gutjnl-2012-302514d.240

${ }^{1} \mathrm{R} O$ Butcher, ${ }^{*}{ }^{2} \mathrm{~S} J$ Mehta, ${ }^{2} \mathrm{O} \mathrm{F}$ Ahmad, ${ }^{2} \mathrm{~S}$ Stein, ${ }^{2} \mathrm{~A}$ Ignjatovic, ${ }^{1} \mathrm{~L} \mathrm{E}$ Loo, ${ }^{1} \mathrm{~A}$ M Abbasi, ${ }^{1} \mathrm{R}$ George, ${ }^{1} \mathrm{R}$ Prudham, ${ }^{2} \mathrm{R}$ Vega, ${ }^{2} \mathrm{~S}$ McCartney, ${ }^{2} \mathrm{~S}$ L Bloom, ${ }^{1} \mathrm{~J} \mathrm{~K}$ Limdi. ${ }^{1}$ Department of Gastroenterology, Pennine Acute Hospitals, Manchester, UK ${ }^{2}$ Department of Gastroenterology, University College London Hospital, London, UK

Introduction The UK Bowel Cancer Screening Programme (BCSP) was launched in 2006 and rolled out in successive waves covering the entire population of England and Wales. It screens individuals aged 60-69 years with a Faecal Occult Blood test (FOBt) followed by a screening colonoscopy if FOBt positive. Our study aimed to quantify the incidental diagnosis of Inflammatory Bowel Disease (IBD) through BCSP and patient outcome in this cohort.

Methods We conducted a retrospective review of BCSP outcomes at our centres from launch in February 2007 until September 2011. Screening data included the number of patients invited, number screened (FOBt outcome "normal" or "abnormal") and number of 
colonoscopies performed. In those with newly diagnosed IBD at colonoscopy confirmed on histology, clinical data including demographics, disease characteristics, treatment and outcome were obtained from case note and electronic patient record review.

Results Of 378424 patients invited, 172244 were screened, representing an uptake of $45.52 \%$ and FOBt positivity of $2.71 \%$. Colonoscopy was performed in 4195 patients (female 1761). Polyps were detected in 1870 (40.14\%), cancer in 279 (5.99\%) and 1216 (26.10\%) had a normal examination. 83 patients had endoscopic appearance suggestive of IBD, confirmed at histology in 44 . Seven patients were excluded as the diagnosis of colitis preceded the screening examination on case note review. Eleven of 37 incidental cases were female. Median age at diagnosis was 64. Twelve patients had Crohn's disease (CD), 22 ulcerative colitis (UC) and three had IBDtype unclassified (IBDU). 31 patients had follow-up data available with a mean follow-up period of 24.4 months. Fifteen patients $(48.4 \%)$ were asymptomatic at diagnosis. Mean values for CRP were 11.8, $\mathrm{Hb}$ 13.8, Platelets 278.5, and Albumin 42.9. Treatment included steroids (8), 5-ASA (25), immunomodulators (azathioprine 5; methotrexate 1) and anti-TNF (infliximab 2; adalimumab 1). None required surgery. Those requiring immunomodulators and/or antiTNF therapy (male 4; female 1) had asymptomatic extensive UC, symptomatic left sided UC, symptomatic left-sided IBDU, symptomatic Crohn's colitis and symptomatic stricturing terminal ileal $\mathrm{CD}$ at diagnosis.

Conclusion An incidental diagnosis of IBD is not uncommon and with the advent of bowel cancer screening this number is set to increase. These patients may present an important model for study of early disease with novel insights and evolving treatment paradigms.

Competing interests None declared.

\section{PWE-241 VITAMIN D STATUS IN INFLAMMATORY BOWEL DISEASE: ARE CLINICIANS SEEING THE LIGHT?}

doi:10.1136/gutjnl-2012-302514d.241

R 0 Butcher, ${ }^{*}$ L E Loo, E Nixon, X McFarlane, J K Limdi. Department of Gastroenterology, Pennine Acute Hospitals, Manchester, UK

Introduction There has been resurgent interest in recent years in the pro-hormone vitamin $\mathrm{D}$ beyond its classical role in bone metabolism recognising its plausible effects in immune regulation. The aim of our study was to review practice relating to vitamin $\mathrm{D}$ assessment among IBD patients.

Methods We conducted a retrospective review of 300 consecutive patients attending IBD clinics at our hospital. Clinical data including demographics, disease characteristics and therapy were obtained from case note and electronic patient record review. Measurement of serum 25-hydroxyvitamin D (25-OHD) concentration subsequent to IBD diagnosis was noted.

Results Of 300 IBD patients reviewed 141 were female. The median age was 47.5 (range 16-91) and mean disease duration 9.6 years. 137 patients had Crohn's disease, 152 patients ulcerative colitis and 11 patients had IBD-type unclassified (IBDU). 126 (42.0\%) patients were current or ex-smokers. Vitamin D status was assessed in 45 $(15.0 \%)$ patients. In 41 patients $(91.1 \%)$ measurement was undertaken within the last 2 years. The mean and median serum $25-\mathrm{OHD}$ level was 19.1 and $17.0 \mathrm{ng} / \mathrm{ml}$ respectively (range 5-49.9). Nine $(20.0 \%)$ of these patients had levels $<10 \mathrm{ng} / \mathrm{ml}$ consistent with deficiency and 17 (37.8\%) levels $<20 \mathrm{ng} / \mathrm{ml}$. Of the nine patients with vitamin $\mathrm{D}$ deficiency (three males; six females), six had Crohn's disease and three had ulcerative colitis. Of the Crohn's patients, three had ileo-colonic, two colonic and one ileal disease. Three had non-stricturing and non-penetrating disease, two stricturing and one penetrating disease. Of the ulcerative colitis patients one had extensive disease, one left-sided disease and one had proc- titis. All patients had received steroids during the course of their disease and three patients received azathioprine, four anti-TNF (three infliximab; one adalimumab) and four had previous surgery. Patients with vitamin $\mathrm{D}$ deficiency had significant disease requiring immunomodulator, anti-TNF therapy and surgery in this cohort.

Conclusion Vitamin D assessment in IBD patients is suboptimal. Hypovitaminosis D is under-recognised and consequently undertreated. The myriad emerging roles of vitamin $\mathrm{D}$ in the pathogenesis of IBD emphasise the importance of recognition and optimisation of vitamin $\mathrm{D}$ status to above $30 \mathrm{ng} / \mathrm{ml}$ in this patient group.

Competing interests None declared.

\section{PWE-242 ACCESSING INFORMATION ON IBD: A SURVEY OF SOURCES AND RELIABILITY OF INTERNET RESOURCES}

doi:10.1136/gutjnl-2012-302514d.242

R A Ley Greaves, * L F Wilkinson, T Orchard. Imperial College, London, UK

Introduction Crohn's and Ulcerative Colitis (UC) are chronic diseases, often requiring lifelong treatment. They can be variable therefore accurate and useful patient information is vital. With an increasing number of sources this can be a cause of confusion for patients. As part of a service evaluation this study examined the information given to patients by doctors, other sources used, how they chose their information and their accuracy.

Methods 100/113 patients agreed to answer a short survey. Topics were: Sources of information, internet usage, checks made on information and reasons for choices. Separately an internet search was performed with three major search engines using five search terms (IBD, UC, Crohn's, Ulcerative colitis and inflammatory bowel disease). From the top 10 hits on each 22 websites that were relevant to the search term were then checked against 5 criteria for accreditation of information.

Results Of 100 patients (43 male, 57 female) with an average age of 44 (18-81). There were 47 Crohn's and 53 UC patients. $65 \%$ had suffered for over 5 years. $48 \%$ wanted more information than given in clinic and $91 \%$ had sought further information. Of those who sought further information $90 \%$ used the internet, $55 \%$ patient leaflets and $25 \%$ used books. Sources used varied with age, $78 \%$ of over 65 s didn't use the internet while only $6 \%$ of under 30 s doing research didn't. Leaflet use in the under 30s was $48 \%$ while being $78 \%$ in the over $65 \mathrm{~s}$. Using the search terms in Google, Yahoo and bing an average of 1, 1.5 and 2 sponsored advertising links were highest ranked respectively. $67 \%$ of those using the internet picked websites on their search engine ranking with $20 \%$ picking on recommendation. $40 \%$ made no checks on the information they found whereas $23 \%$ said they "read around". Of the 22 relevant websites 7 filled 5 accreditation criteria and 6 scored 2 or less. Crohn's and Colitis UK (NACC) website scored highly for accreditation and $45 \%$ of patients used it $62 \%$ of whom had been recommended it by their doctor. However its mean search engine ranking was 7 th and it only appeared in the top ten in 9/12 searches. In the free text section a number of patients commented on concerns about inaccurate information.

Conclusion The results show patient information is mainly sourced from the internet and patient leaflets. Internet information is of varying quality and search engine rankings are influenced by sponsored links directed at IBD patients as opposed to accredited sites. Data on use of the NACC website with and without recommendation show simple measures such as a health care professional taking time to advise a patient may direct them to towards better information and away from lower quality potentially misleading sites. Many patients commented that websites with unsubstantiated information caused them significant concern.

Competing interests None declared. 